\title{
THE RANK OF A HARDY FIELD ${ }^{1}$
}

BY

\author{
MAXWELL ROSENLICHT
}

\begin{abstract}
A Hardy field is a field of germs of real-valued functions on positive half-lines that is closed under differentiation. Its rank is the rank of the associated ordered abelian group, the value group of the canonical valuation of the field. The properties of this rank are worked out, its relevance to asymptotic expansions indicated, examples provided, and applications given to the order of growth of solutions of ordinary differential equations.
\end{abstract}

1. Introduction. This paper concerns the behavior near $+\infty$ of real-valued functions on positive half-lines and their possible asymptotic expansions there, including expansions that are possibly more refined than the usual rank 1 Poincare power series in $1 / x$. For example, for any unbounded $y(x)$ on $(0,+\infty)$ such that $y^{\prime \prime}=x^{x} y$, one has the rank 3 expansion

$$
\frac{y^{\prime}}{y} \sim x^{x / 2}-\frac{1+\log x}{4}+\left(\frac{1}{8 x}-\frac{(1+\log x)^{2}}{32}\right) x^{-x / 2}+\cdots .
$$

For this and all other results quoted in this section, we refer to [9].

We work in the context of Hardy fields. A Hardy field is a set of germs at $+\infty$ of real-valued functions on positive half-lines which is closed under differentiation and which forms a field under ordinary addition and multiplication. Examples of Hardy fields are any subfield of $\mathbf{R}$, the field of rational functions of one variable $\mathbf{R}(x)$, and any $L$-field of Hardy ( $L$ is for logarithmico-exponential), an $L$-field being a field of (germs of) functions obtained from $\mathbf{R}(x)$ by repeated adjunctions of real-valued algebraic functions, logarithms of positive functions, and exponentials of functions (e.g. the field $\left.\mathbf{R}\left(x, \sqrt{\log x}, e^{x}, \exp \left(x \sqrt{\log x}+e^{x}\right)\right)\right)$. More generally, if $k$ is a Hardy field and $f$ a germ such that $f$ is algebraic over $k$ or $f^{\prime} \in k$ or $f^{\prime} / f \in k$, then $k(f)$ is a Hardy field.

First results on Hardy fields say that any element of a Hardy field is ultimately (i.e., for sufficiently large $x \in \mathbf{R}$ ) of constant sign,+ 0 , or - , and that $\lim _{x \rightarrow+\infty} f(x)$ exists as an element of $\mathbf{R} \cup\{+\infty,-\infty\}$. Equally fundamental is the following description of the "canonical valuation" $\nu$ of a Hardy field.

Received by the editors November 1, 1982. Most of the contents of this paper were presented at a meeting of the American Mathematical Society at Madison, Wisconsin , on April 16, 1982.

1980 Mathematics Subject Classification. Primary 34E05, 41A60; Secondary 12H05, 13N05, 26 A12.

'Research supported by National Science Foundation grant number MCS-8102055.

$$
\begin{aligned}
& \text { (c) } 1983 \text { American Mathematical Society } \\
& 0002-9947 / 83 \$ 1.00+\$ .25 \text { per page }
\end{aligned}
$$


THEOREM 0. Let $k$ be a Hardy field. Then there exists a map $\nu$ from the set of nonzero elements $k^{*}$ of $k$ onto an ordered abelian group such that:

(1) if $a, b \in k^{*}$, then $\nu(a b)=\nu(a)+\nu(b)$;

(2) if $a \in k^{*}$, then $\nu(a) \geqslant 0$ if and only if $\lim _{x \rightarrow+\infty} a(x) \in \mathbf{R}$;

(3) writing symbolically $\nu(0)=+\infty$, if $a, b \in k$ then $\nu(a+b) \geqslant \min \{\nu(a), \nu(b)\}$, with equality if $\nu(a) \neq \nu(b)$;

(4) if $a, b \in k^{*}$ and $\nu(a), \nu(b) \neq 0$, then $\nu(a) \geqslant \nu(b)$ if and only if $\nu\left(a^{\prime}\right) \geqslant \nu\left(b^{\prime}\right)$;

(5) if $a, b \in k^{*}$ and $\nu(a)>\nu(b) \neq 0$, then $\nu\left(a^{\prime}\right)>\nu\left(b^{\prime}\right)$.

This is Theorem 4 of [9]. According to (1) and (2), if $a \in k^{*}$ then $\nu(a)>0$ if and only if $\lim _{x \rightarrow+\infty} a(x)=0$, while $\nu(a)<0$ if and only if $\lim _{x \rightarrow+\infty} a(x)= \pm \infty$. The set $\left\{\nu(a): a \in k^{*}\right\}$ is the value group of $k$. The easy proof of the theorem starts with the definition of the value group as the set of equivalence classes of elements of $k^{*}$, where $a, b \in k^{*}$ are called equivalent if and only if $\lim _{x \rightarrow+\infty} a(x) / b(x) \in \mathbf{R}^{*}$. Parts (4), (5) are consequences of l'Hospital's rule.

COROllaRy. Writing $a \sim b$ whenever $a, b \in k^{*}$ and $\lim _{x \rightarrow+\infty} a(x) / b(x)=1$ :

(1) $\sim$ is an equivalence relation;

(2) if $a \sim b$ and $c \sim d$, then $a c \sim b d$ :

(3) if $a, b \in k^{*}$, then $a \sim b$ if and only if $\nu(a-b)>\nu(a)$;

(4) if $a, b \in k^{*}, a \sim b$, and $\nu(a)(=\nu(b)) \neq 0$, then $a^{\prime} \sim b^{\prime}$;

(5) if $a \in k$ and $\nu(a)=0$, then $a \sim \lim _{x \rightarrow+\infty} a(x)$.

It should be remarked that much of this can be done in the more general context of differential valuations [7].

\section{Asymptotic integration.}

Proposition 1. If $k$ is a Hardy field and $a, b \in k^{*}$, with $\nu(a) \geqslant 0$ and $\nu(b) \neq 0$, then $\nu\left(a^{\prime}\right)>\nu\left(b^{\prime} / b\right)$.

We may assume $\nu(a)=0$, since we may otherwise replace $a$ by $a+1$. As $x \rightarrow+\infty$ we have $a(x), b(x)$ approaching a nonzero real number and 0 or $\pm \infty$, respectively, so that l'Hospital's rule implies $a=a b / b \sim\left(a b^{\prime}+a^{\prime} b\right) / b^{\prime}=a+$ $a^{\prime} b / b^{\prime}$, so that by (3) of the above corollary we have $\nu\left(a^{\prime} b / b^{\prime}\right)>\nu(a)$, or $\nu\left(a^{\prime}\right)>$ $\nu\left(b^{\prime} / b\right)$.

A consequence of the last result is that if the element $a$ of a Hardy field $k$ satisfies $\nu(a) \geqslant 0$, then $\nu\left(a^{\prime}\right)>0$. For $k(x)$ is a Hardy field, so $\nu\left(a^{\prime}\right)>\nu\left(x^{\prime} / x\right)=\nu(1 / x)>$ 0 . Thus if the value group $\nu\left(k^{*}\right)$ is $\{0\}$, we have $k \subset \mathbf{R}$.

Proposition 2. Let $k$ be a Hardy field and let $\Psi=\left\{\nu\left(u^{\prime} / u\right): u \in k^{*}, \nu(u) \neq 0\right\}, a$ subset of the value group. If l.u.b. $\Psi$ exists, it is not of the form $\nu\left(g^{\prime}\right)$ for any $g \in k^{*}$ such that $\nu(g) \neq 0$.

Suppose the contrary. Then $\nu\left(g^{\prime} / g\right) \in \Psi$, so $\nu\left(g^{\prime}\right) \geqslant \nu\left(g^{\prime} / g\right)$, so $\nu(g) \geqslant 0$, so $\nu(g)>0$. Suppose, for a moment, there exists a $g_{1} \in k^{*}$ such that $\nu(g)>\nu\left(g_{1}\right)>0$. Then $\nu\left(g^{\prime}\right)>\nu\left(g_{1}^{\prime}\right)$. Since $\nu\left(g^{\prime}\right)=$ l.u.b. $\Psi, \nu\left(g_{1}^{\prime}\right)$ is not an upper bound for $\Psi$, which contradicts Proposition 1. Thus $\nu(g)$ is the smallest positive element of the 
value group $\nu\left(k^{*}\right)$. If $u \in k^{*}$ and $\nu(u) \neq 0$ then $\nu\left(g^{\prime}\right)>\nu\left(u^{\prime} / u\right)$, so $\nu\left(g^{\prime} / g\right) \geqslant$ $\nu\left(u^{\prime} / u\right)$, or $\nu\left(g^{\prime} / g\right) \geqslant$ l.u.b. $\Psi$. Since $\nu\left(g^{\prime} / g\right)<\nu\left(g^{\prime}\right)$, we have a contradiction to l.u.b. $\Psi=\nu\left(g^{\prime}\right)$.

Proposition 1 implies that the set $\Psi$ is always bounded from above. If $\mathbf{R} \subset k$, then $\nu\left(g^{\prime}\right) \neq$ l.u.b. $\Psi$ for any $g \in k$; we need only check this for $\nu(g)=0$, in which case there exists $c \in \mathbf{R}$ such that $\nu(g-c)>0$, so $\nu\left(g^{\prime}\right)=\nu\left((g-c)^{\prime}\right) \neq$ l.u.b. $\Psi$.

The following result and the later Proposition 4, which together constitute principle $(*)$ of [7], are proved here for the convenience of the reader. We call an element $a$ of a Hardy field infinitely increasing if $\lim _{x \rightarrow+\infty} a(x)=+\infty$.

Proposition 3. If $k$ is a Hardy field and $a, b \in k^{*}$, with $|\nu(a)| \geqslant|\nu(b)|>0$, in particular if $a$ and $b$ are infinitely increasing and $a \geqslant b$, then $\nu\left(b^{\prime} / b\right) \geqslant \nu\left(a^{\prime} / a\right)$.

Without altering $|\nu(a)|,|\nu(b)|, \nu\left(a^{\prime} / a\right), \nu\left(b^{\prime} / b\right)$, we can replace $a$ and $b$, respectively, by $\pm a$ or $\pm 1 / a$ and $\pm b$ or $\pm 1 / b$ to reduce to the case where $a$ and $b$ are infinitely increasing. Then $|\nu(a)|=\nu(1 / a),|\nu(b)|=\nu(1 / b)$, so $\nu(b) \geqslant \nu(a)$, or $b<M a$, for some positive number $M$, for $x$ sufficiently large. Then $\log b<\log a+$ $\log M$. Since $a, b$ are infinitely increasing, we get $\nu(\log b) \geqslant \nu(\log a)$, so (4) or (5) of Theorem 0 implies $\nu\left(b^{\prime} / b\right) \geqslant \nu\left(a^{\prime} / a\right)$.

LEMMA. Let $k$ be a Hardy field and $f \in k^{*}$, with $\nu(f) \neq l . u . b . \Psi, \Psi$ being the set $\left\{\nu\left(u^{\prime} / u\right): u \in k^{*}, \nu(u) \neq 0\right\}$. Then there exists $u_{0} \in k^{*}$ with $\nu\left(u_{0}\right) \neq 0$ such that if $u \in k^{*}$ and $\left|\nu\left(u_{0}\right)\right| \geqslant|\nu(u)|>0$, then $|\nu(u)| \leqslant\left|\nu\left(f u / u^{\prime}\right)\right|$.

We can exclude the case $\nu\left(k^{*}\right)=\{0\}$, for then $\Psi=\varnothing$ and l.u.b. $\Psi=0$. First suppose $\nu(f)$ is not an upper bound for $\Psi$. Then we first choose $u_{1} \in k^{*}$ such that $\nu\left(u_{1}\right) \neq 0$ and $\nu(f)<\nu\left(u_{1}^{\prime} / u_{1}\right)$, then choose $u_{0} \in k^{*}$ such that

$$
\left|\nu\left(u_{0}\right)\right|=\min \left\{\left|\nu\left(u_{1}\right)\right|,\left|\nu\left(f u_{1} / u_{1}^{\prime}\right)\right|\right\} .
$$

Then if $u \in k^{*}$ and $\left|\nu\left(u_{0}\right)\right| \geqslant|\nu(u)|>0$, we have $\nu\left(u^{\prime} / u\right) \geqslant \nu\left(u_{0}^{\prime} / u_{0}\right) \geqslant \nu\left(u_{1}^{\prime} / u_{1}\right)$, by Proposition 3 , so $\nu(f)<\nu\left(u^{\prime} / u\right)$ and

$$
\left|\nu\left(f u / u^{\prime}\right)\right|=\nu\left(u^{\prime} / f u\right) \geqslant \nu\left(u_{1}^{\prime} / f u_{1}\right)=\left|\nu\left(f u_{1} / u_{1}^{\prime}\right)\right| \geqslant\left|\nu\left(u_{0}\right)\right| \geqslant|\nu(u)| .
$$

If, on the other hand, $\nu(f)$ is an upper bound for $\Psi$, then by assumption it is not a least upper bound, so there exists $f_{1} \in k^{*}$ such that $\nu(f)>\nu\left(f_{1}\right)$ and $\nu\left(f_{1}\right)$ is an upper bound for $\Psi$. Then if $u \in k^{*}$ and $\nu(u) \neq 0$, we have $\nu\left(f u / u^{\prime}\right)=\nu\left(f / f_{1}\right)+$ $\nu\left(f_{1} u / u^{\prime}\right) \geqslant \nu\left(f / f_{1}\right)>0$, so the demands of the lemma are satisfied if we take $u_{0}=f / f_{1}$.

THEOREM 1. Let $k$ be a Hardy field and $f \in k^{*}$, with $\nu(f) \neq$ l.u.b. $\Psi$, where $\Psi=\left\{\nu\left(u^{\prime} / u\right): u \in k^{*}, \nu(u) \neq 0\right\}$. Then there exists $u_{0} \in k^{*}$ with $\nu\left(u_{0}\right) \neq 0$ such that if $u \in k^{*}$ and $\left|\nu\left(u_{0}\right)\right| \geqslant|\nu(u)|>0$, then

$$
\left(f \cdot\left(f u / u^{\prime}\right) /\left(f u / u^{\prime}\right)^{\prime}\right)^{\prime} \sim f .
$$


Choose $u_{0}$ as in the lemma. Then $u^{\prime} \neq 0$, for otherwise $u \in \mathbf{R}^{*}$ and $\nu(u)=0$. Therefore $f u / u^{\prime}$ exists. If $\left(f u / u^{\prime}\right)^{\prime}=0$, then $f u / u^{\prime} \in \mathbf{R}^{*}$, giving $\nu\left(f u / u^{\prime}\right)=0$, a contradiction of the lemma. Therefore

$$
\left(f \cdot \frac{\left(f u / u^{\prime}\right)}{\left(f u / u^{\prime}\right)^{\prime}}\right)^{\prime}=\left(\frac{f}{\left(f u / u^{\prime}\right)^{\prime}} \cdot\left(f u / u^{\prime}\right)\right)^{\prime}=f+\left(f u / u^{\prime}\right)\left(\frac{f}{\left(f u / u^{\prime}\right)^{\prime}}\right)^{\prime}
$$

so we have to show that $\nu\left(\left(f /\left(f u / u^{\prime}\right)^{\prime}\right)^{\prime}\right)>\nu\left(u^{\prime} / u\right)$ whenever $\left|\nu\left(u_{0}\right)\right| \geqslant|\nu(u)|>0$. This being so, the lemma implies $\left|\nu\left(f u / u^{\prime}\right)\right| \geqslant|\nu(u)|>0$, so by Proposition 3 we have

$$
\nu\left(u^{\prime} / u\right) \geqslant \nu\left(\left(f u / u^{\prime}\right)^{\prime} /\left(f u / u^{\prime}\right)\right), \quad \text { or } \quad \nu\left(f /\left(f u / u^{\prime}\right)^{\prime}\right) \geqslant 0,
$$

so Proposition 1 implies $\nu\left(\left(f /\left(f u / u^{\prime}\right)^{\prime}\right)^{\prime}\right)>\nu\left(u^{\prime} / u\right)$.

A related result, much weaker for Hardy fields, is [8, Theorem 2].

3. Comparability and rank. Nonzero elements $\alpha, \beta$ of an ordered abelian group are called comparable if there are positive integers $m, n$ such that $m|\alpha|>|\beta|$ and $n|\beta|$ $>|\alpha|$. This is clearly an equivalence relation on the set of nonzero elements of the ordered abelian group. In the same way, nonzero elements $f, g$ of a Hardy field $k$ are called comparable if $\nu(f), \nu(g) \neq 0$ and $\nu(f)$ and $\nu(g)$ are comparable in the value group $\nu\left(k^{*}\right)$. Thus the term "comparable" can be applied only to nonzero elements of $k$ whose limits as $x \rightarrow+\infty$ are 0 or $\pm \infty$. Comparability is an equivalence relation among the elements $f$ of $k^{*}$ such that $\nu(f) \neq 0$, and we speak of comparability classes of such elements. For any $f \in k^{*}$ such that $\nu(f) \neq 0$, the elements $\pm f$, $\pm 1 / f$ are in the same comparability class, and precisely one of these is infinitely increasing. If $f, g \in k$ are infinitely increasing, then either $f \leqslant g$ or $g \leqslant f$ (ultimately), and if $f \leqslant g$ then $f$ and $g$ are comparable if and only if there is a positive integer $N$ such that $g \leqslant f^{N}$. We call the comparability class of $f$ greater than that of $g$ if, $f$ and $g$ being infinitely increasing, $f$ is greater than any power of $g$. If $f, g \in k^{*}$ are comparable and $\nu(f), \nu(g)$ have the same sign, then $f$ and $g$ are both comparable to $f g$. If $f, g \in k^{*}$, with $\nu(f), \nu(g) \neq 0$, and the comparability class of $f$ is greater than that of $g$, then $f g$ is comparable to $f$. For any $f \in k^{*}$ with $\nu(f) \neq 0$ and any nonzero real number $c, \pm|f|^{c}$ is comparable to $f$ (working in a Hardy field extension of $k$ ). If $f, g \in k^{*}$ are comparable and $f$ is infinitely increasing, then there is a unique $c \in \mathbf{R}^{*}$ such that for each real number $\varepsilon>0$ we have $f^{c-\varepsilon}<|g|<f^{c+\varepsilon}$, and either $\nu\left(g / f^{c}\right)$ $=0$ or the comparability class of $g / f^{c}$ is smaller than that of $f$.

Proposition 4. Let $k$ be a Hardy field, $a, b \in k^{*}, \nu(a), \nu(b) \neq 0$, and $\nu\left(a^{\prime} / a\right)=$ $\nu\left(b^{\prime} / b\right)$. Then $a$ and $b$ are comparable.

Without loss of generality we may suppose $a$ and $b$ are infinitely increasing and $a \geqslant b$. Then $\log a, \log b$ are infinitely increasing, $\log a \geqslant \log b$, and $\log a / \log b$ has the same limit as $x \rightarrow \infty$ as $(\log a)^{\prime} /(\log b)^{\prime}=\left(a^{\prime} / a\right) /\left(b^{\prime} / b\right)$, a nonzero real number. Thus for some positive integer $N$ we have $\log a / \log b<N$, or $a<b^{N}$.

We define the rank of a Hardy field $k$ to be the number of comparability classes of $k$, denoting this $\mathrm{rk} k$. By Propositions 3 and 4 , rk $k=\operatorname{card} \Psi$, where $\Psi=$ $\left\{\nu\left(u^{\prime} / u\right): u \in k^{*}, \nu(u) \neq 0\right\}$. The Hardy field $\mathbf{R}\left(x, \log x, e^{x}\right)$ has rank 3 , with $x$, 
$\log x$ and $e^{x}$ representatives of its comparability classes. A Hardy field $k$ has rank zero if and only if $k \subset \mathbf{R}$. As a consequence of the following result, most Hardy fields arising in practice have finite rank.

Proposition 5. Let $k \subset K$ be Hardy fields. If $t_{1}, \ldots, t_{n} \in K^{*}$ are algebraically dependent over $k$, then there are integers $a_{1}, \ldots, a_{n}$, not all zero, such that $\nu\left(t_{1}^{a_{1}} \cdots t_{n}^{a_{n}}\right)$ $\in \nu\left(k^{*}\right)$. In particular, if $r$ is the transcendence degree $r=\operatorname{deg} \operatorname{tr} K / k$, then there are at most $r$ comparability classes of $K$ that do not have representatives in $k$, so rk $K \leqslant$ rk $k+r$.

The first part of this is easily proved by writing down a polynomial relation among $t_{1}, \ldots, t_{n}$ with coefficients in $k$ and noting that when $\nu$ is applied to the nonzero terms, at least two of these must give the same result. For the rest it suffices to note that if $t_{1}, \ldots, t_{n} \in K^{*}$ are in distinct comparability classes and $a_{1}, \ldots, a_{n}$ are nonzero integers, then $t_{1}^{a_{1}} \cdots t_{n}^{a_{n}}$ is in the largest of these comparability classes.

A Hardy field $k$ will have finite rank if it is of finite transcendence degree over its subfield of constants. Such is the case if $k=\mathbf{R}\langle y\rangle\left(=\mathbf{R}\left(y, y^{\prime}, y^{\prime \prime}, \ldots\right)\right.$, the smallest field closed under differentiation that contains $\mathbf{R}$ and $y$ ) and $y$ satisfies an algebraic differential equation (ADE) with coefficients in $k$. For if $y$ is a solution of such an $\operatorname{ADE}$ of order $n$, then $y^{(n)}$ is algebraic over $\mathbf{R}\left(y, y^{\prime}, \ldots, y^{(n-1)}\right)$. Differentiating an irreducible polynomial relation with coefficients in $k$ among $y, y^{\prime}, \ldots, y^{(n)}$ shows that $y^{(n+1)} \in \mathbf{R}\left(y, y^{\prime}, \ldots, y^{(n)}\right)$, hence $y^{(n+2)}, y^{(n+3)}, \ldots \in \mathbf{R}\left(y, y^{\prime}, \ldots, y^{(n)}\right)$ and $k=$ $\mathbf{R}\langle y\rangle=\mathbf{R}\left(y, y^{\prime}, \ldots, y^{(n)}\right)$. Here $\operatorname{deg} \operatorname{tr} k / \mathbf{R} \leqslant n$.

Lemma. Let $k$ be a Hardy field, $f, g \in k-\mathbf{R}, \min \{\nu(f), \nu(g)\} \neq 0$, and $f^{\prime} \sim g^{\prime}$. Then $f \sim g$.

Assume $\nu(f) \leqslant \nu(g)$. By (3) of the corollary to Theorem 0 , we must show that $\nu(f-g)>\nu(f)$. If not, then $\nu(f-g) \leqslant \nu(f)$, which produces the false result $\nu\left(f^{\prime}-g^{\prime}\right) \leqslant \nu\left(f^{\prime}\right)$, unless $\nu(f-g)=0$. Since $\nu(g) \geqslant \nu(f) \neq 0$, we must have $\nu(f), \nu(g)<0$, contradicting $\nu(f-g) \leqslant \nu(f)$.

Proposition 6. Let $k$ be a Hardy field, let $f \in k$ be infinitely increasing, and suppose $k$ has comparability classes smaller than that of $f$. Then there exists $g \in k$ such that $g \sim \log f$.

Let $h$ be an infinitely increasing element of $k$ whose comparability class is smaller than that of $f$. By Propositions 3 and $4, \nu\left(h^{\prime} / h\right)>\nu\left(f^{\prime} / f\right)$, so $\nu\left(f^{\prime} / f\right) \neq 1$.u.b. $\Psi$, where $\Psi$ is as before. Theorem 1 gives the existence of $g \in k$ such that $g^{\prime} \sim f^{\prime} / f$. In a larger Hardy field, $f^{\prime} / f=(\log f)^{\prime}$ and $\nu(\log f)<0$. By Proposition $1, \nu(g)<0$. By the lemma, $g \sim \log f$.

We recall the standard notation according to which, for $n \in \mathbf{Z}$, the function $l_{n}$ is defined on a positive half-line by $l_{n}(x)=\log ^{n} x$, the superscript here indicating functional composition, so that $l_{-1}(x)=e^{x}, l_{0}(x)=x, l_{1}(x)=\log x, l_{2}(x)=$ $\log \log x$. As usual, the function $e_{n}$ is defined by $e_{n}(x)=l_{-n}(x)$.

THEOREM 2. Let $k$ be a Hardy field of finite positive rank $n$. Let $w$ be an infinitely increasing element of the largest comparability class of $k$. If $k$ contains an element $u_{0} \sim x$, then there exist nonnegative integers $r, s$, with $r+s+1 \leqslant n$, and a positive 
real number $A$ such that $k$ contains elements $u_{0}, u_{1}, \ldots, u_{r}$, with each $u_{i} \sim l_{i}(x)$, such that $u_{r}$ is in the smallest comparability class of $k$, and such that $w<e_{s}\left(x^{A}\right)$. If $k$ contains no $u_{0} \sim x$, then for some positive integer $s \leqslant n, k$ contains an element $u \sim l_{s-1}(w)$ that is contained in the smallest comparability class of $k$, and we have $u^{\prime} / u \sim c$, a positive real number, so for any real $\varepsilon>0$ we have

$$
e^{(c-\varepsilon) x}<u<e^{(c+\varepsilon) x} \text { and } e_{s}((c-\varepsilon) x)<w<e_{s}((c+\varepsilon) x) .
$$

$k$ contains infinitely increasing elements $w_{0}, w_{1}, w_{2}, \ldots$, with $w_{0}=w, w_{1} \sim \log w_{0}$, $w_{2} \sim \log w_{1}, \ldots$, representatives of a strictly decreasing sequence of comparability classes of $k$, the sequence terminating with a representative of the smallest comparability class of $k$. If $k$ contains a $u_{0} \sim x$, then for some least $s \geqslant 0$ the comparability class of $w_{s}$ will be at most that of $x$, so $w_{s}<x^{A_{0}}$ for some positive real $A_{0}$, giving $l_{s}(w)<x^{A}$ if $A>A_{0}$, hence $w<e_{s}\left(x^{A}\right)$. Similarly, $k$ contains $u_{0} \sim x, u_{1} \sim$ $l_{1}(x), \ldots, u_{r} \sim l_{r}(x)$, for some $r \geqslant 0$, with $u_{r}$ in the smallest comparability class of $k$. If $s>0$, then $w=w_{0}, w_{1}, \ldots, w_{s-1}, u_{0}, \ldots, u_{r}$ are mutually incomparable, if $s=0$ the elements $u_{0}, \ldots, u_{r}$, so in either case we have $r+s+1 \leqslant n$. Now suppose $k$ contains no $u_{0} \sim x$, that is, no $u_{0}$ such that $u_{0}^{\prime} \sim 1$. By Theorem $1, \max \Psi=0$. Therefore for the last element of the sequence $w_{0}=w, w_{1} \sim \log w, w_{2} \sim \log w_{1}, \ldots$, say $u=w_{s-1} \sim l_{s-1}(w)$, we have $\nu\left(u^{\prime} / u\right)=0$, so $u^{\prime} / u \sim c$, a positive real number. Clearly $1 \leqslant s \leqslant n$ and $l_{s}(w) \sim \log u \sim c x$, so we are done.

$\mathbf{R}(x)$ and $\mathbf{R}\left(e^{x}\right)$ are the simplest examples of Hardy fields of rank 1. More interesting is the case $k=\mathbf{Q}\langle y\rangle$, where $y:(0,+\infty) \rightarrow(0,+\infty)$ is given by $y(0)=1$, $y^{\prime}=\sqrt{2+1 / y}$. This is a Hardy field since $k=\mathbf{Q}\left(y^{\prime}\right)$, each element of which is ultimately of constant sign as $x \rightarrow+\infty$, for then $y \rightarrow+\infty$ and $y^{\prime} \rightarrow \sqrt{2}$. We have $y^{\prime} \sim \sqrt{2}, y \sim \sqrt{2} x, y / y^{\prime} \in k, y / y^{\prime} \sim x, r=s=0, n=1$, and $\mathbf{Q}$ is the field of constants of $k$. The field $k_{1}=\mathbf{Q}\langle z\rangle$, where $z=\cosh \sqrt{2} x$, is a Hardy field since $k_{1}=\mathbf{Q}\left(z, z^{\prime}\right)$, with $z^{2}-\left(z^{\prime}\right)^{2} / 2=1$, is a subfield closed under differentiation of the Hardy field $\mathbf{R}\left(e^{\sqrt{2} x}\right)$. In the case of $k_{1}$ we have rank 1 with $z$ infinitely increasing and $z^{\prime} / z \sim \sqrt{2} . k_{1}$ contains no element $\sim x, s=n=1$, and again the subfield of constants of $k_{1}$ is $\mathbf{Q}$. An example of a Hardy field with $r+s+1<n$ is given by $k_{3}=\mathbf{R}(x, \sqrt{\log x}, \exp (\sqrt{\log x}))$. Here $x, \exp (\sqrt{\log x}), \log x$ are representatives of the $n=3$ comparability classes in decreasing order since $\exp (\sqrt{\log x})<\exp (\log x)=x$, and $r=1, s=0$.

THEOREM 3. Let $k \subset K$ be Hardy fields, where $K$ contains at most $n$ elements that are mutually incomparable and incomparable to any element of $k$. Also suppose $k$ contains a smallest comparability class. Then $K$ contains a smallest comparability class and there are integers $r, s \geqslant 0$ satisfying $r+s \leqslant n$ such that the smallest comparability class of $K$ contains an element $\sim l_{r}(f)$ for any infinitely increasing $f$ in the smallest comparability class of $k$, and for any given $t \in K$ there exists $g \in k$ such that $t<e_{s}(g)$.

The proof is similar to the proof of Theorem 2, but simpler. Take $f$ an infinitely increasing element of the smallest comparability class of $k$. If $K$ contains smaller comparability classes than that of $f$, then $K$ contains an element $\sim \log f$, if $K$ contains smaller comparability classes than that of the last element, then $K$ contains 
an element $\sim l_{2}(f)$, etc. Similarly, if $t$ is an infinitely increasing element of $K$ then, if the comparability class of $t$ is greater than each comparability class of $k, K$ contains an element $\sim \log t$, if the comparability class of this last element is greater than each comparability class of $k$ then $K$ contains an element $\sim l_{2}(t)$, etc.

Results on the order of growth of solutions of ADEs which overlap with Theorems 2 and 3, but which are on the whole rather weaker, have been proved by different methods in [10,2 and 11].

4. Rational rank. Examples. If $k \subset K$ are Hardy fields there is a natural order-preserving embedding of ordered abelian groups $\nu\left(k^{*}\right) \subset \nu\left(K^{*}\right)$. By Proposition 5, if $u \subset K^{*}$ is algebraic over $k$, then some nonzero integral multiple of $\nu(u)$ is in $\nu\left(k^{*}\right)$. If $K$ is taken to include all $N$ th roots of all positive elements of $k$, then each element of $\nu\left(k^{*}\right)$ is divisible in $\nu\left(K^{*}\right)$ by each $N \neq 0$, and therefore $\nu\left(k^{*}\right)$ is a subgroup of an ordered vector space over $\mathbf{Q}$. Denote the ordered rational vector space containing $\nu\left(k^{*}\right)$ by $\mathbf{Q} \nu\left(k^{*}\right)$. The vector space dimension $\operatorname{dim}_{\mathbf{Q}} \mathbf{Q} \nu\left(k^{*}\right)$ is called the rational rank of $k$; this is the cardinality of any maximal subset of $k^{*}$ any nontrivial integral power product of whose elements has nonzero $\nu$-value. The rational rank of $k$ is at least the rank. By Proposition 5 the rational rank of $k$ is at most its transcendence degree over its subfield of constants. The Hardy field $\mathbf{R}\left(x, x^{\pi}\right)$ has rank 1 and rational rank 2 .

We discuss asymptotic expansions in a Hardy field $k \supset \mathbf{R}$ of finite rational rank $n$. Let $t_{1}, \ldots, t_{n} \in k$ be infinitely increasing elements such that $\nu\left(t_{1}\right), \ldots, \nu\left(t_{n}\right)$ are linearly independent over $\mathbf{Q}$. If $u \in k^{*}$, then $u \sim c t_{1}^{a_{1}} \cdots t_{n}^{a_{n}}$ for certain unique $c \in \mathbf{R}^{*}$ and $a_{1}, \ldots, a_{n} \in \mathbf{Q}$, and the same is true if $u$ is a nonzero element of the extension field of $k$ obtained by adjoining all $N$ th roots of positive elements, since this extension field also has rational rank $n$. Writing $u-c t_{1}^{a_{1}} \cdots t_{n}^{a_{n}} \sim d t_{1}^{b_{1}} \cdots t_{n}^{b_{n}}$, with $d \in \mathbf{R}^{*}, b_{1}, \ldots, b_{n} \in \mathbf{Q}$, we have

$$
\nu(u)=\nu\left(t_{1}^{a_{1}} \cdots t_{n}^{a_{n}}\right)<\nu\left(t_{1}^{b_{1}} \cdots t_{n}^{b_{n}}\right),
$$

and we can continue this process to get an asymptotic expansion (possibly terminating)

$$
u \sim c t_{1}^{a_{1}} \cdots t_{n}^{a_{n}}+d t_{1}^{b_{1}} \cdots t_{n}^{b_{n}}+\cdots .
$$

Suppose $t_{1}>t_{2}>\cdots>t_{n}$. If for certain $i, j$, such that $1 \leqslant i \leqslant j \leqslant n$, we had $t_{i}, t_{i+1}, \ldots, t_{j}$ comparable but some certain integral power product of these in a smaller comparability class, then we could delete one of $t_{i}, t_{i+1}, \ldots, t_{j}$ in the sequence $t_{1}, t_{2}, \ldots, t_{n}$, replacing it later in the sequence by the certain power product, to get a similar sequence but with fewer $t$ 's in the comparability class of $t_{i}$. Continuing this process, we can get $t_{1}, \ldots, t_{n}$ to be of the form $t_{11}, t_{12}, \ldots, t_{\ln (1)}$, $t_{21}, \ldots, t_{2 n(2)}, \ldots, t_{r 1}, \ldots, t_{r n(r)}$, where $n=n(1)+\cdots+n(r), t_{11}>t_{12}>\cdots>t_{r n(r)}$, each set $\left\{t_{11}, t_{12}, \ldots, t_{1 n(1)}\right\},\left\{t_{21}, \ldots, t_{2 n(2)}\right\}, \ldots,\left\{t_{r 1}, \ldots, t_{r n(r)}\right\}$ consists of comparable elements and no nontrivial integral power product of the elements of any one set is in a smaller comparability class. Clearly $r=$ rk $k$. For integers $a_{11}, \ldots, a_{r n(r)}$, not all zero, the sign of $\nu\left(t_{11}^{a_{11}} \cdots t_{\ln (1)}^{\left.a_{\ln (1)} \cdots t_{r n(r)}^{a_{r(r)}}\right)}\right.$ is the same as that of $\nu\left(t_{i 1}^{a_{i 1}} \cdots t_{i n(i)}^{\left.a_{i n(i)}\right)}\right)$ if $i$ is the smallest positive integer such that $a_{i 1}, \ldots, a_{i n(i)}$ are not all zero. For each 
$i=1, \ldots, r$, the subgroup of $\nu\left(k^{*}\right)$ generated by $\nu\left(t_{i 1}\right), \ldots, \nu\left(t_{i n(i)}\right)$ is order-isomorphic to a subgroup of $\mathbf{R}$, so there exist $c_{i 1}, \ldots, c_{i n(i)} \in \mathbf{R}$, linearly independent over $\mathbf{Q}$, such that for all $a_{i 1}, \ldots, a_{i n(i)} \in \mathbf{Q}$ the sign of $\nu\left(t_{i 1}^{a_{i 1}} \cdots t_{i n(i)}^{\left.a_{i n(i)}\right)}\right.$ is the same as that of $a_{i 1} c_{i 1}+\cdots+a_{i n(i)} c_{i n(i)}$. Finally, for $i=1, \ldots, r-1$ we have

$$
\left.\log t_{i 1} \sim c_{i} \cdot \text { (rational power product of } t_{i+1,1}, \ldots, t_{r n(r)}\right) \text {, }
$$

with $c_{i}$ a positive real number and the rational power product of negative $\nu$-value that is strictly increasing with $i$. In the special case where the value group $\nu\left(k^{*}\right)$ is finitely generated, we may choose $t_{11}, \ldots, t_{r n(r)}$ to be a set of generators of this group, in which case all the above rational exponents are integers. In the nicest case of all, where $\nu\left(k^{*}\right)$ has $r=$ rk $k$ generators $\nu\left(t_{1}\right), \ldots, \nu\left(t_{r}\right)$, each $n(i)=1$ and the elements of $k$ admit asymptotic expansions in real multiples of integral power products of $t_{1}, \ldots, t_{r}$.

Let $k \supset \mathbf{R}$ be a Hardy field of rank $n$ and let $t_{1}>t_{2}>\cdots>t_{n}$ be infinitely increasing representatives of the various comparability classes of $k$. Suppose that if we adjoin to $k$ any set of real powers of $t_{1}, \ldots, t_{n}$ then the resulting Hardy field has the same rank $n$ as $k$. Then for each nonzero $u \in k$ (or $u \in$ smallest extension field of $k$ containing all real powers of $t_{1}, \ldots, t_{n}$ ), we have $u \sim c t_{1}^{a_{1}} \cdots t_{n}^{a_{n}}$, with unique real $c, a_{1}, \ldots, a_{n}$, with $c \neq 0$, so that the elements of $k$ admit asymptotic expansions in terms of real multiples of products of real powers of $t_{1}, \ldots, t_{n}$.

The author has announced earlier (unpublished) an incorrect result to the effect that if $k$ is a Hardy field, $t$ a positive element of $k$, and $c \in k \cap \mathbf{R}$, then $k$ and $k\left(t^{c}\right)$ have the same rank. For a counterexample, take $k=\mathbf{R}(x, \sqrt{\log x}, y)$, where $y=$ $\exp (\pi \log x+\sqrt{\log x})=x^{\pi} \exp (\sqrt{\log x})$. If $m, n, p$ are integers not all zero, then $\nu\left(y^{m} x^{n}(\sqrt{\log x})^{p}\right)=\nu\left(x^{m \pi+n} \exp (m \sqrt{\log x})(\log x)^{p / 2}\right) \neq 0$. It follows that the value group $\nu\left(k^{*}\right)$ is generated by $\nu(y), \nu(x)$, and $\nu(\sqrt{\log x})$, and there are only two comparability classes, those of $x$ and of $\log x$. Thus $k$ has rational rank 3 and rank 2 . But $k\left(x^{\pi}\right)=k\left(x, \sqrt{\log x}, x^{\pi}, \exp (\sqrt{\log x})\right)$ has rank 3 . The correct general results here will appear in another paper; these affirm, among other things, that a Hardy field of finite rational rank that contains $\mathbf{R}$ is a subfield of a Hardy field of the type occurring in the last paragraph.

Now consider the field $\mathbf{R}\langle\Gamma(x)\rangle$ of real meromorphic functions on the positive real line consisting of all rational functions with real coefficients of the gamma function and all its derivatives. We shall prove that this field (or, rather, the corresponding set of germs of functions with values in $\mathbf{R} \cup\{\infty\}$ as $x \rightarrow+\infty$ ) is a Hardy field of rank 3 , a result that has been proved quite differently by Boshernitzan [3]. To simplify the exposition we first give a short proof of Hölder's theorem that $\Gamma(x)$ satisfies no ADE, by an argument close to the original one [5] and almost the same as that in the recent proof [1].

LEMMA. $\Gamma(x)$ is not a solution of any ADE with coefficients in $\mathbf{R}(x)$.

This is equivalent to showing that $\operatorname{deg} \operatorname{tr} \mathbf{R}\left(x, \Gamma(x), \Gamma^{\prime}(x), \ldots\right) / \mathbf{R}$ is infinite. Setting

$$
\phi(x)=\Gamma^{\prime}(x) / \Gamma(x) \text { for } x>0,
$$


it suffices to prove that $\operatorname{deg} \operatorname{tr} \mathbf{R}\left(\phi^{\prime}(x), \phi^{\prime \prime}(x), \ldots\right) / \mathbf{R}$ is infinite. As with all other proofs of Hölder's theorem, the present one is based on the fact that since $\Gamma(x+1)=x \Gamma(x)$, we have $\phi(x+1)=\phi(x)+1 / x$, hence $\phi^{(n)}(x+1)=\phi^{(n)}(x)$ $+(-1)^{n} n ! x^{-n-1}$ for $n=0,1,2, \ldots$ We have to show, for any positive integer $n$, the nonexistence of a nonzero $F\left(x, X_{1}, \ldots, X_{n}\right) \in \mathbf{R}(x)\left[X_{1}, \ldots, X_{n}\right]$, where $X_{1}, \ldots, X_{n}$ are indeterminates, such that $F\left(x, \phi^{\prime}(x), \ldots, \phi^{(n)}(x)\right)=0$. Suppose the contrary. If we choose such an $F\left(x, X_{1}, \ldots, X_{n}\right)$ such that $n$ is minimal, then such that the total degree of $F\left(x, X_{1}, \ldots, X_{n}\right)$ in $X_{1}, \ldots, X_{n}$ is minimal (which last condition implies the polynomial $F\left(x, X_{1}, \ldots, X_{n}\right)$ is irreducible), then such that the coefficient of a specific one of its terms of maximal total degree is 1 , we get $F\left(x, X_{1}, \ldots, X_{n}\right)$ unique. Since $F\left(x, \phi^{\prime}(x), \ldots, \phi^{(n)}(x)\right)=0$, we have $F\left(x+1, \phi^{\prime}(x+1), \ldots, \phi^{(n)}(x+1)\right)=0$, or

$$
F\left(x+1, \phi^{\prime}(x)-x^{-2}, \ldots, \phi^{(n)}(x)+(-1)^{n} n ! x^{-n-1}\right)=0 .
$$

By the uniqueness property of $F$ we get

$$
F\left(x+1, X_{1}-x^{-2}, \ldots, X_{n}+(-1)^{n} n ! x^{-n-1}\right)=F\left(x, X_{1}, \ldots, X_{n}\right) .
$$

Thus there exists a nonconstant $G\left(x, X_{1}, \ldots, X_{n}\right) \in \mathbf{R}(x)\left[X_{1}, \ldots, X_{n}\right]$ such that

$$
G\left(x+1, X_{1}-x^{-2}, \ldots, X_{n}+(-1)^{n} n ! x^{-n-1}\right)=G\left(x, X_{1}, \ldots, X_{n}\right) .
$$

We shall show this to be impossible, thereby completing the proof. We cannot have $G\left(x, X_{1}, \ldots, X_{n}\right) \in \mathbf{R}(x)$, for then we would have a $g(x) \in \mathbf{R}(x)-\mathbf{R}$ such that $g(x+1)=g(x)$, which is impossible since the finite nonempty set of zeros and poles of $g(x)$ in $\mathbf{C}$ would have to be invariant under translation by 1 . The set of all $G\left(x, X_{1}, \ldots, X_{n}\right) \in \mathbf{R}(x)\left[X_{1}, \ldots, X_{n}\right]$ such that

$$
G\left(x, X_{1}-x^{-2}, \ldots, X_{n}+(-1)^{n} n ! x^{-n-1}\right)=G\left(x, X_{1}, \ldots, X_{n}\right)
$$

is closed under the operators $\partial / \partial X_{1}, \ldots, \partial / \partial X_{n}$. Hence there exists a $G\left(x, X_{1}, \ldots, X_{n}\right)$ of the form $a(x) X_{i}+b(x)$, for some $a(x), b(x) \in \mathbf{R}(x)$ such that $a(x) \neq 0$, and some $i=1, \ldots, n$. From the equation

$$
a(x+1)\left(X_{i}+(-1)^{i} i ! x^{-i-1}\right)+b(x+1)=a(x) X_{i}+b(x)
$$

follows $a(x+1)=a(x)$, so $a \in \mathbf{R}^{*}$, then $(-1)^{i} a i ! x^{-i-1}+b(x+1)=b(x)$. For any $c \in \mathbf{C}^{*}$, both $c$ and $c+1$ are poles of $b(x)$ or neither is. Since $b(x)$ has only a finite number of poles, this number is zero, so $b(x)$ is a polynomial, which is clearly impossible.

THEOREM 4. $\mathbf{R}\langle\Gamma(x)\rangle$ is a Hardy field of rank 3.

We begin with the Stirling development (cf. [4, p. 180 or 6, p. 294])

$$
\log \Gamma(x) \sim\left(x-\frac{1}{2}\right) \log x-x+\frac{1}{2} \log 2 \pi+\sum_{s=1}^{\infty} \frac{B_{2 s}}{2 s(2 s-1) x^{2 s-1}},
$$


where the $B_{2 s}$ are the Bernoulli numbers and $\sim$ is to be interpreted in the classical way, and the consequent expansions

$$
\begin{gathered}
\phi(x) \sim \log x-\frac{1}{2 x}-\sum_{s=1}^{\infty} \frac{B_{2 s}}{2 s} x^{-2 s}, \\
\phi^{\prime}(x) \sim \sum_{s=0}^{\infty} B_{s} x^{-1-s}, \\
\phi^{(n)}(x) \sim \sum_{s=0}^{\infty}(-1)^{n-1} \frac{(s+n-1) !}{s !} B_{s} x^{-n-s} .
\end{gathered}
$$

Actually, we do not use these expansions, only the fact that $\phi^{\prime}(x), \phi^{\prime \prime}(x), \ldots$ have asymptotic expansions in increasing powers of $1 / x$. Consider the formal power series field

$$
\mathbf{R}((1 / x))=\left\{\sum_{i=0}^{\infty} a_{i} x^{m-i}: m \in \mathbf{Z}, a_{0}, a_{1}, a_{2}, \ldots \in \mathbf{R}\right\}
$$

and its elements $\bar{\phi}^{(n)}(x), n=1,2,3, \ldots$, given by the asymptotic expansions of $\phi^{(n)}(x)$, so $\bar{\phi}^{(1)}(x)=\sum_{s=0}^{\infty} B_{s} x^{-1-s}$ and for each $n=1,2, \ldots, \bar{\phi}^{(n+1)}(x)=\left(\bar{\phi}^{(n)}(x)\right)^{\prime}$. We deduce from $\phi^{(n)}(x+1)=\phi^{(n)}(x)+(-1)^{n} n ! x^{-n-1}$ that

$$
\bar{\phi}^{(n)}(x+1)=\bar{\phi}^{(n)}(x)+(-1)^{n} n ! x^{-n-1} .
$$

It follows that $\bar{\phi}^{(1)}(x), \bar{\phi}^{(2)}(x), \ldots$ are algebraically independent over the subfield $\mathbf{R}(x)$ of $\mathbf{R}((1 / x))$, for otherwise there would exist a nonzero expression $F\left(x, X_{1}, \ldots, X_{n}\right) \in \mathbf{R}(x)\left[X_{1}, \ldots, X_{n}\right], X_{1}, \ldots, X_{n}$ being indeterminates, such that $F\left(x, \bar{\phi}^{(1)}(x), \ldots, \bar{\phi}^{(n)}(x)\right)=0$, and the proof of the lemma could be followed, simply replacing each $\phi$ by $\bar{\phi}$, to produce a contradiction. Thus for each nonzero differential polynomial $H(Y) \in \mathbf{R}\left[Y, Y^{\prime}, Y^{\prime \prime}, \ldots\right]$ ( $Y$ being a differential indeterminate) we have $H\left(\bar{\phi}^{(1)}(x)\right) \neq 0$, which implies $H\left(\phi^{\prime}(x)\right) \sim a x^{-i}$ for some real nonzero $a$ and some nonnegative integer $i$. In particular, $H\left(\phi^{\prime}(x)\right)$ is of constant sign, + or - , for sufficiently large $x$, so the germ of $H\left(\phi^{\prime}(x)\right)$ on positive half-lines is invertible. Therefore $\mathbf{R}\left\langle\phi^{\prime}(x)\right\rangle$ is a Hardy field and we have an embedding $\mathbf{R}\left\langle\phi^{\prime}(x)\right\rangle \rightarrow \mathbf{R}((1 / x))$ given by $\phi^{(n)}(x) \mapsto \bar{\phi}^{(n)}(x)$. Note that $\phi^{\prime}(x) \sim 1 / x$. The field $\mathbf{R}\langle\phi(x)\rangle$, obtained from $\mathbf{R}\left\langle\phi^{\prime}(x)\right\rangle$ by adjoining an antiderivative, is also a Hardy field, of rank 2 , since $\phi(x) \sim \log x$. The field $\mathbf{R}\langle\Gamma(x)\rangle=(\mathbf{R}\langle\phi(x)\rangle)(\Gamma(x))$, obtained from $\mathbf{R}\langle\phi(x)\rangle$ by the adjunction of an exponential of an integral of $\phi(x)$, is also a Hardy field, this time of rank 3 , since $\Gamma(x)$ exceeds any power of $x$.

COROLlaRY. The value group of the Hardy field

$$
\mathbf{R}\left\langle x, \log x, \exp \left(\left(x-\frac{1}{2}\right) \log x-x\right), \Gamma(x)\right\rangle
$$

is generated by $\nu(\Gamma(x))\left(=\nu\left(\exp \left(\left(x-\frac{1}{2}\right) \log x-x\right)\right)\right), \nu(x)$, and $\nu(\log x)$.

The indicated field is a Hardy field, since it is an extension of a known Hardy field by antiderivatives and exponentials. To prove the rest, define

$$
u(x)=\Gamma(x) / \sqrt{2 \pi} \exp \left(\left(x-\frac{1}{2}\right) \log x-x\right)
$$


so

$$
\log u \sim \sum_{s=1}^{\infty} B_{2 s} / 2 s(2 s-1) x^{2 s-1} .
$$

Hence $u(x) \sim \bar{u}(x)$, where $\bar{u}(x) \in \mathbf{R}((1 / x))$ is given by

$$
\bar{u}(x)=\exp \left(\sum_{s=1}^{\infty} B_{2 s} / 2 s(2 s-1) x^{2 s-1}\right) .
$$

Suppose for a moment that $\bar{u}(x), \bar{u}^{\prime}(x), \bar{u}^{\prime \prime}(x), \ldots$ are algebraically dependent over $\mathbf{R}(x)$. Then the subfield $\mathbf{R}\langle x, \bar{u}(x)\rangle$ of $\mathbf{R}((1 / x))$ is of finite transcendence degree over $\mathbf{R}(x)$. Since $u^{\prime}(x) / u(x)=\phi(x)-\log x+1 / 2 x$, we have $\left(u^{\prime}(x) / u(x)\right)^{\prime}=$ $\phi^{\prime}(x)-1 / x-1 / 2 x^{2}$, and therefore $\left(\bar{u}^{\prime}(x) / \bar{u}(x)\right)^{\prime}=\bar{\phi}^{(1)}(x)-1 / x-1 / 2 x^{2}$. Thus $\bar{\phi}^{(1)}(x) \in \mathbf{R}\langle x, \bar{u}(x)\rangle$ so $\mathbf{R}\left\langle x, \bar{\phi}^{(1)}(x)\right\rangle$ has finite transcendence degree over $\mathbf{R}(x)$, which contradicts an earlier result. Therefore $\bar{u}(x), \bar{u}^{\prime}(x), \bar{u}^{\prime \prime}(x), \ldots$ are algebraically independent over $\mathbf{R}(x)$. We deduce that the Hardy field $\mathbf{R}\langle x, u(x)\rangle$ is differentially isomorphic to the differential subfield $\mathbf{R}\langle x, \bar{u}(x)\rangle$ of $\mathbf{R}((1 / x))$ and the value group of $\mathbf{R}\langle x, u(x)\rangle$ is generated by $\nu(x)$. In the Hardy field $\mathbf{R}\langle\log x, u(x)\rangle=$ $(\mathbf{R}\langle x, u(x)\rangle)(\log x), \log x$ is of smaller comparability class than $x, \operatorname{so} \mathbf{R}\langle\log x, u(x)\rangle$ has value group generated by $\nu(x)$ and $\nu(\log x)$. The Hardy field

$$
\mathbf{R}\left\langle x, \log x, \exp \left(\left(x-\frac{1}{2}\right) \log x-x\right), \Gamma(x)\right\rangle=(\mathbf{R}\langle\log x, u(x)\rangle)(\Gamma(x))
$$

contains the still larger comparability class of $\Gamma(x)$, so its value group is generated by $\nu(x), \nu(\log x)$, and $\nu(\Gamma(x))$.

We now consider the field $\mathbf{R}\langle\zeta(x)\rangle$ of real meromorphic functions on the positive line consisting of all rational functions with real coefficients of the Riemann zeta function $\zeta(x)$ and all its derivatives. We identify the elements of this field with the corresponding germs of functions with values in $\mathbf{R} \cup\{\infty\}$ as $x \rightarrow+\infty$.

PROPOSITION 7. $\mathbf{R}\langle\zeta(x)\rangle$ is a Hardy field of rank 1 with value group generated by $\left\{\nu\left(p^{x}\right): p\right.$ a prime number $\}$. The function $\zeta(x)$ is not a solution of any ADE with coefficients in $\mathbf{R}$.

We shall actually prove much more. Consider the set $R$ of Dirichlet series of the form $\sum_{n=1}^{\infty} a_{n} / n^{x}$, where each $a_{n} \in \mathbf{R}$ and $\left|a_{n}\right|<M n^{r}$, for certain real $M, r$ depending only on the series. Each series converges to a real analytic function on some positive half-line. An easy estimate shows that for the above series and for any positive integer $m$ we have $\left|\Sigma_{n>m} a_{n} / n^{x}\right|=o\left(1 / m^{x}\right)$ as $x \rightarrow+\infty$, so for $x$ sufficiently large the sign of the series is the sign of its first nonzero coefficient. The set $R$, or the set of functions, or germs on positive half-lines, represented by the series of $R$, is a ring closed under differentiation and its field of quotients is a Hardy field. For the series $f(x)=\sum_{n>1} a_{n} / n^{x}$ and any integer $m \geqslant 1$ we have

$$
f^{(m)}(x)=\sum_{n>1}(-\log n)^{m} a_{n} / n^{x} .
$$

It follows that a suitable real linear combination of $f(x), f^{\prime}(x), \ldots, f^{(m-1)}(x)$ will be a Dirichlet series whose first term is $a_{m} / m^{x}$. Therefore the value group of $\mathbf{R}\langle f(x)\rangle$ 
is generated by $\left\{\nu\left(n^{x}\right): n=2,3, \ldots, a_{n} \neq 0\right\}$. The value group of the full Hardy field of all quotients of elements of $R$ is generated by $\left\{\nu\left(p^{x}\right): p\right.$ a prime number $\}$, therefore it has rank 1. The function $f(x)$ can satisfy an ADE with coefficients in $\mathbf{R}$ only if $\operatorname{deg} \operatorname{tr} \mathbf{R}\langle f(x)\rangle / \mathbf{R}$ is finite, which is true only if $\mathbf{R}\langle f(x)\rangle$ has finite rational rank, a necessary condition for which is that the set of prime numbers dividing the various $n>0$ for which $a_{n} \neq 0$ is finite. For $\zeta(x)$, where each $a_{n}=1$, the quoted results are immediate.

A slight modification of the last train of ideas gives another interesting example. Let $R$ be the set of all formal expressions

$$
a_{0}+\sum_{n, i_{n 1}, i_{n 2} \ldots i_{n n}} a\left(i_{n 1}, i_{n 2}, \ldots, i_{n n}\right)\left(e_{1}(x)\right)^{i_{n 1}} \cdots\left(e_{n}(x)\right)^{i_{n n}},
$$

with $n$ ranging over all positive integers, $i_{n 1}, \ldots, i_{n n}$ over all integers, and $a_{0}$ and each $a\left(i_{n 1}, \ldots, i_{n n}\right) \in \mathbf{R}$, for which there exists some $A \in \mathbf{R}$ such that each $\left|a\left(i_{n 1}, \ldots, i_{n n}\right)\right|$ $\leqslant A^{n}$, each $\left|i_{n j}\right| \leqslant A$, and each $i_{n n}<0$. It is easy to verify that the given series converges uniformly on $(0,+\infty)$ to a real analytic function, which we denote by the same expression, the function approaches the limit $a_{0}$ as $x \rightarrow+\infty$, and if $a_{0}=0$ but the series itself is not zero, then the function differs from that nonzero term $a\left(i_{n 1}, i_{n 2}, \ldots, i_{n n}\right)\left(e_{1}(x)\right)^{i_{n 1}} \cdots\left(e_{n}(x)\right)^{i_{n n}}$ for which $n$ is minimal and $\left(i_{n n}, \ldots, i_{n 2}, i_{n 1}\right)$ maximal in the lexicographical ordering by a fraction of the latter term that approaches 0 as $x \rightarrow+\infty$, so the function has a constant sign, + or - , for $x$ sufficiently large. The set of all such functions or, rather, germs of such as $x \rightarrow+\infty$, is an integral domain closed under differentiation whose field of quotients is a Hardy field whose value group is generated by $\left\{\nu\left(e_{n}(x)\right): n=1,2,3, \ldots\right\}$.

Proposition 8. If $y=\sum_{n=1}^{\infty}\left(1 / e_{n}(x)\right)$ then $\mathbf{R}\langle y\rangle$ is a Hardy field whose value group is generated by $\left\{\nu\left(e_{n}(x)\right): n=1,2,3, \ldots\right\}$, and $y$ is not the solution of any $A D E$ with coefficients in $\mathbf{R}$.

As a subfield closed under differentiation of a Hardy field, $\mathbf{R}\langle y\rangle$ is a Hardy field. We claim that if $F\left(Y_{0}, \ldots, Y_{m}\right) \in \mathbf{R}\left[Y_{0}, \ldots, Y_{m}\right], Y_{0}, \ldots, Y_{m}$ being indeterminates, and $F \neq 0$, then $F\left(y, y^{\prime}, \ldots, y^{(m)}\right) \neq 0$. For suppose $F\left(y, y^{\prime}, \ldots, y^{(m)}\right)=0$ and for $n=$ $1,2,3, \ldots$ set $y_{n}=\sum_{i=1}^{n} 1 / e_{i}(x)$. Then the terms of the expansion of $F\left(y_{n}, y_{n}^{\prime}, \ldots, y_{n}^{(m)}\right)$ dominate all terms involving $e_{n+1}(x), e_{n+2}(x), \ldots$ in $F\left(y, y^{\prime}, \ldots, y^{(m)}\right)$, and therefore $F\left(y_{n}, y_{n}^{\prime}, \ldots, y_{n}^{(m)}\right)=0$. That is,

$$
F\left(y_{n-1}+1 / e_{n}(x), \ldots, y_{n-1}^{(m)}+\left(1 / e_{n}(x)\right)^{(m)}\right)=0 .
$$

Note that each of $1 / e_{n}(x),\left(1 / e_{n}(x)\right)^{\prime}, \ldots,\left(1 / e_{n}(x)\right)^{(m)}$ is of the form $\left(1 / e_{n}(x)\right)$. (polynomial with integer coefficients in $e_{1}(x), e_{2}(x), \ldots, e_{n-1}(x)$ ). Letting $G\left(Y_{0}, \ldots, Y_{m}\right) \in \mathbf{R}\left[Y_{0}, \ldots, Y_{m}\right]$ be the homogeneous component of $F$ of maximal total degree, we get $G \neq 0$ but $G\left(1 / e_{n}(x),\left(1 / e_{n}(x)\right)^{\prime}, \ldots,\left(1 / e_{n}(x)\right)^{(m)}\right)=0$ (for $e_{1}(x), e_{2}(x), \ldots$ are algebraically independent over $\mathbf{R}$, since they are mutually incomparable). Therefore

$$
\operatorname{deg} \operatorname{tr} \mathbf{R}\left\langle e_{n}(x)\right\rangle / \mathbf{R}=\operatorname{deg} \operatorname{tr} \mathbf{R}\left\langle 1 / e_{n}(x)\right\rangle / \mathbf{R} \leqslant m,
$$


which is a contradiction if $n>m$, since $\operatorname{rk} \mathbf{R}\left\langle e_{n}(x)\right\rangle \geqslant n$ by Theorem 2 . Therefore $y$ is not a solution of any ADE with coefficients in $\mathbf{R}$. For any $n$ we have $y_{n} \in$ $\mathbf{R}\left(e_{1}(x), \ldots, e_{n}(x)\right)$, a field of transcendence degree $n$ over $\mathbf{R}$, so $\operatorname{deg} \operatorname{tr} \mathbf{R}\left\langle y_{n}\right\rangle / \mathbf{R} \leqslant n$, and therefore $y_{n}, y_{n}^{\prime}, \ldots, y_{n}^{(n)}$ are algebraically dependent over $\mathbf{R}$. Therefore there exists a nonzero $H\left(Y_{0}, \ldots, Y_{n}\right) \in \mathbf{R}\left[Y_{0}, \ldots, Y_{n}\right]$ such that $H\left(y_{n}, y_{n}^{\prime}, \ldots, y_{n}^{(n)}\right)=0$. But $H\left(y, y^{\prime}, \ldots, y^{(n)}\right) \neq 0$, and the expansion of $H\left(y, y^{\prime}, \ldots, y^{(n)}\right)$ in terms of power products of $e_{1}(x), e_{2}(x), \ldots$ involves no terms involving only $e_{1}(x), e_{2}(x), \ldots, e_{n}(x)$. Therefore $H\left(y, y^{\prime}, \ldots, y^{(n)}\right) \in \mathbf{R}\langle y\rangle$ is comparable to $e_{N}(x)$ for some $N>n$. Therefore the value group of $\mathbf{R}\langle y\rangle$ contains

$$
\nu\left(\left(e_{N}(x)\right)^{\prime} / e_{N}(x)\right)=\nu\left(e_{1}(x) e_{2}(x) \cdots e_{N-1}(x)\right) .
$$

Now $e_{1}(x) e_{2}(x) \cdots e_{N-1}(x)$ is comparable to $e_{N-1}(x)$, so the value group of $\mathbf{R}\langle y\rangle$ contains

$$
\nu\left(\left(e_{N-1}(x)\right)^{\prime} / e_{N-1}(x)\right)=\nu\left(e_{1}(x) e_{2}(x) \cdots e_{N-2}(x)\right),
$$

and therefore also $\nu\left(e_{N-1}(x)\right)$, therefore also $\nu\left(e_{N-2}(x)\right)$, etc. Thus the value group of $\mathbf{R}\langle y\rangle$ contains $\nu\left(e_{n}(x)\right)$ for each $n$, and we are done.

ACKNOWLEDGEMENT. The author is grateful for a number of suggestions of the referee.

\section{REFERENCES}

1. S. Bank and R. Kaufman, A note on Hölder's theorem concerning the gamma function, Math. Ann. 232 (1978), 115-120

2. M. Boshernitzan, New "orders of infinity", J. Analyse Math. (to appear).

3. ___ "Orders of infinity" generated by difference equations, Amer. J. Math. (to appear).

4. N. Bourbaki, Fonctions d'une variable réele. Chapitre VII ("La Fonction Gamma"), 2nd ed., Hermann, Paris, 1961.

5. O. Hölcier, Über die Eigenschaft der Г-Funktion, keiner algebraischen Differentialgleichung zu genügen, Math. Ann. 28 (1887), 1-13.

6. F. Olver, Asymptotics and special functions, Academic Press, New York, 1974.

7. M. Rosenlicht, Differential valuations, Pacific J. Math. 65 (1976), 301-319.

8. __ On the value group of a differential valuation, Amer. J. Math. 101 (1979), 258-266.

9. Hardy fields, J. Math. Anal. Appl. 93 (1983), 297-311.

10. M. Singer, Asymptotic behavior of solutions of differential equations and Hardy fields, preliminary report, SUNY at Stony Brook, 1976 (unpublished).

11. L. van den Dries, Bounding the rate of growth of solutions of algebraic differential equations and exponential equations in Hardy fields (to appear).

Department of Mathematics, University of California, Berkeley, California 94720 